\title{
Photostability of liquid crystals and alignment layers
}

\author{
Chien-Hui Wen \\ Sebastian Gauza \\ Shin-Tson Wu
}

\begin{abstract}
Photostability of liquid-crystal (LC) materials and surface alignment layers was evaluated using a UV lamp and a blue laser beam. Both organic polyimide (PI) and inorganic silicon-dioxide $\left(\mathrm{SiO}_{2}\right)$ alignment layers were studied under nitrogen environment. Two commercial TFT-grade LC mixtures (low-birefringence MLC-9200-000 and high-birefringence TL-216) were used for comparisons. Results indicate that $\mathrm{SiO}_{2}$ alignment layers are much more robust than PI layers, and low birefringence LCs are more stable than the high-birefringence ones. At the He-Cd laser wavelength $(\lambda=442 \mathrm{~nm})$, both LC mixtures and $\mathrm{SiO}_{2}$ alignment layers are hardly damaged. To lengthen the lifetime of an LCD projector, inorganic $\mathrm{SiO}_{2}$ alignment layers, high-optical-density UV filter, long cutoff-wavelength blue filter, and short-conjugation (low birefringence) LC materials should be considered.
\end{abstract}

Keywords — Liquid crystals, photostability, lifetime, alignment layers, inorganic $\mathrm{SiO}_{2}$, LCD projector.

\section{Introduction}

Photostability of liquid-crystal (LC) materials and alignment layers play a crucial role in affecting the lifetime of liquid-crystal-display (LCD) devices. ${ }^{1-4}$ In direct-view displays, ultraviolet (UV) light is often used to seal the LC panels. In projection displays using liquid-crystal-on-silicon (LCOS), ${ }^{5}$ the employed arc lamp is relatively bright. Although a cold mirror and a hot mirror are used to filter out the unwanted UV and infrared contents of the lamp, residual UV light could still penetrate into the LC panels and cause performance degradation. Even if the UV contents are completely filtered out, the blue wavelength might still be harmful to the LCD panels. These high-energy photons could cause gradual degradation of the organic alignment layers and the LC molecules. Once the LC medium is decomposed or the alignment layers deteriorated, the pretilt angle is changed and the consequent electro-optic effects are altered. ${ }^{6}$

In this paper, we report the photostability of LC alignment layers and LC materials in the UV and blue wavelengths. Regarding the alignment layers stability, we studied the buffed polyimide (PI) and sputtered inorganic silicondioxide $\left(\mathrm{SiO}_{2}\right)$ alignment layers under nitrogen environment. On the LC-material stability studies, we selected two Merck LC mixtures: TL-216 $(\Delta n=0.20)$ and MLC-9200$000(\Delta n=0.08)$. A high birefringence LC mixture, such as TL-216, enables a thinner cell gap to be used. The advantages are twofold: (1) faster response time and (2) weaker fringing-field effect. For instance, the Philips color-sequential LCOS projector ${ }^{7}$ uses a $\sim 1-\mu \mathrm{m}$ cell gap in order to achieve fast response time and minimize the fringing-field effect. On the other hand, a low-birefringence LC mixture, such as MLC-9200-000, is commonly used for three-panel LCOS projectors.

\section{UV stability of LC alignment layers}

The Hamamatsu UV light was delivered to the sample through a fiber bundle and collimated by a lens. The output light passed through a narrow-band interference filter centered at $\lambda \sim 365 \mathrm{~nm}$. The light intensity at the sample position was measured to be $I \sim 350 \mathrm{~mW} / \mathrm{cm}^{2}$. Beside the UV stability, we also studied the material stability of TL-216 in $\mathrm{SiO}_{2}$ cell at $\lambda=442 \mathrm{~nm}$. A He-Cd laser $(\lambda=442 \mathrm{~nm})$ was used as the light source to illuminate the LC cell directly. The light intensity at the sample position was controlled to be $20 \mathrm{~W} / \mathrm{cm}^{2}$.

Three kinds of homogeneous LC cells were studied: two with commercial PI layers (PI-A and PI-B) and one with a $\mathrm{SiO}_{2}$ alignment layer. These PI cells were purchased from different venders in order to compare the PI performances.

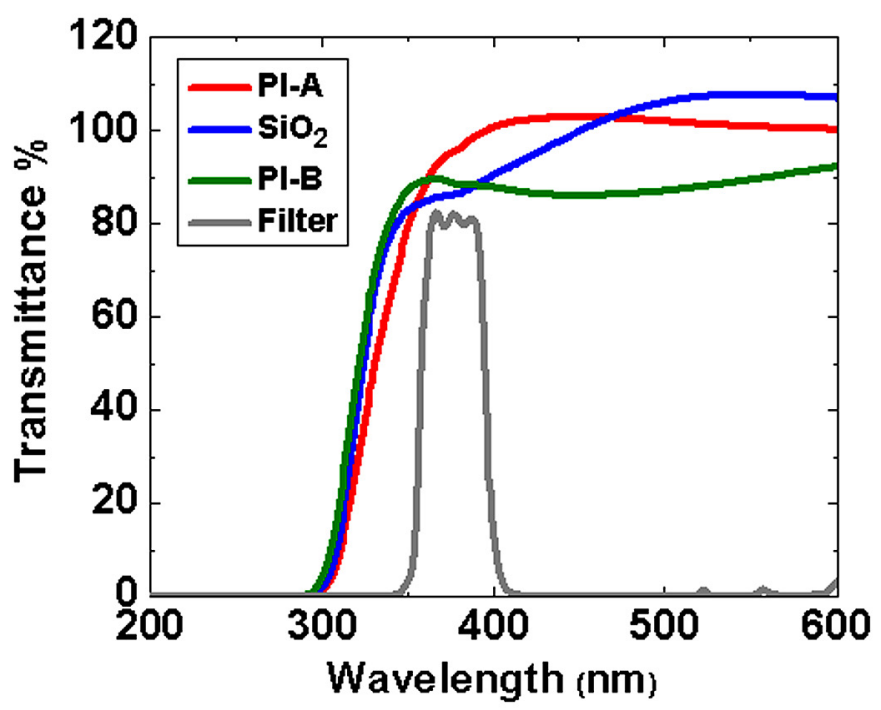

FIGURE 1 - Transmittance of PI-A (red line), $\mathrm{SiO}_{2}$ (blue line), PI-B (green line), and filter (gray line).

Received 6/13/05; accepted 7/23/05.

The authors are with the College of Optics and Photonics, University of Central Florida, Orlando, FL 32816; telephone 407/823-4763, fax -6880, e-mail: swu@mail.ucf.edu.

(c) Copyright 2005 Society for Information Display 1071-0922/05/1309-0805\$1.00 

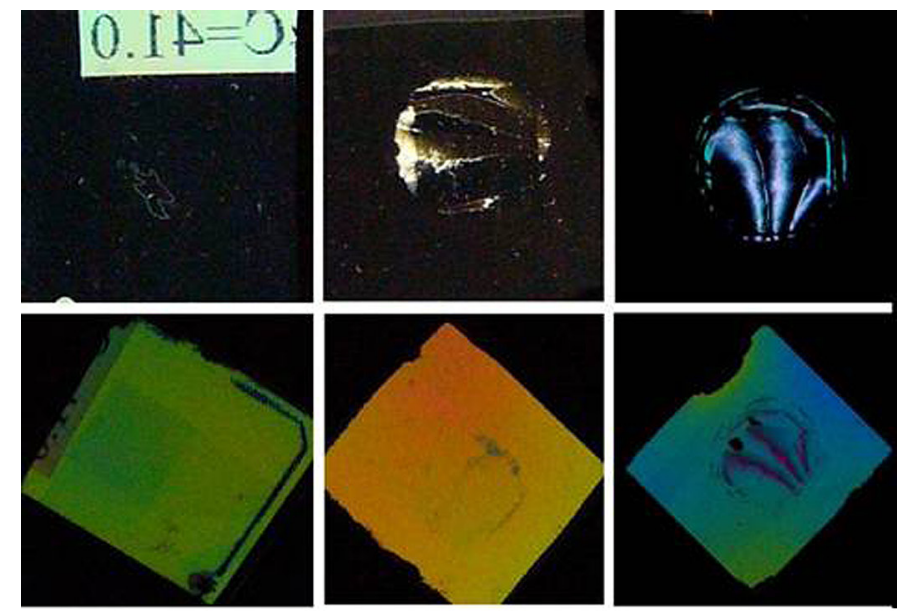

(a)
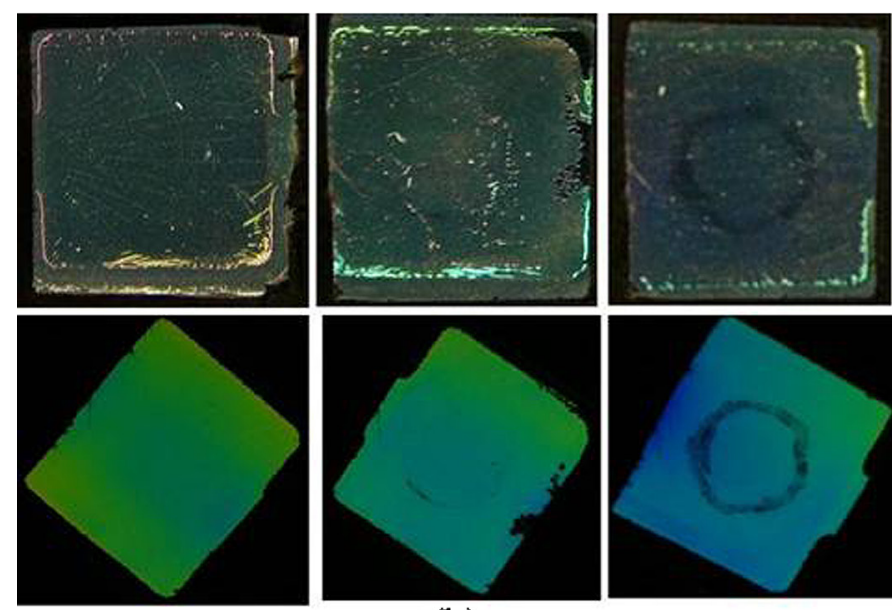

(b)

FIGURE 2 - (a) PI-B and (b) $\mathrm{SiO}_{2}$ alignment cells after 5 (left), 10 (middle), and 15 (right) hours of UV exposures.

Figure 1 shows the measured UV absorption spectra of these cells. Here, red, green, and blue lines represent PI-A, $\mathrm{PI}-\mathrm{B}$, and $\mathrm{SiO}_{2}$ cells, respectively. The transmittance of the UV filter was included as reference. The transmittance of each cell at $\lambda \sim 365 \mathrm{~nm}$ has the following order: PI-A > PI-B $>\mathrm{SiO}_{2}$. In principle, $\mathrm{SiO}_{2}$ alignment layers are highly transparent from $\lambda>200 \mathrm{~nm}$. The absorption of the cells is mainly from the indium-tin-oxide (ITO) electrode. Different cells may have slightly different ITO thickness. As a result, their transmission in the UV region is somewhat different.

In order to compare the UV stability of LC alignment layers, the test cells were covered with a black paper and divided into two areas. The UV light passes through the center of the cell only. These empty cells were illuminated under nitrogen environment. At each stage, we inspected the cell by filling the cell with 5CB (4- $n$-pentyl- $4^{\prime}$-cyanobiphenyl) LC. We observed the cells on a light table between crossed polarizers after 5, 10, and 15 hours of UV illumination. Results are shown in Figs. 2(a) and 2(b). Figures 2(a) and 2(b) show the photos of PI-B and $\mathrm{SiO}_{2}$ cells, respectively. The PI-A has similar results as PI-B.

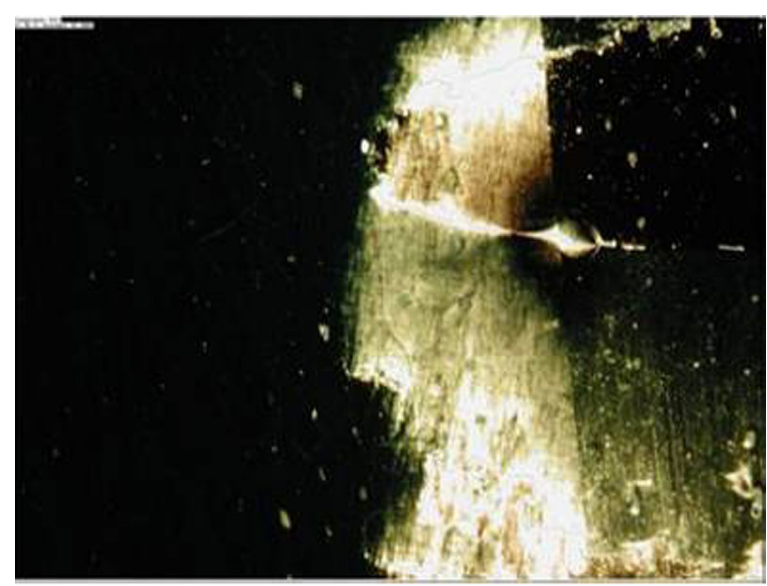

(a)

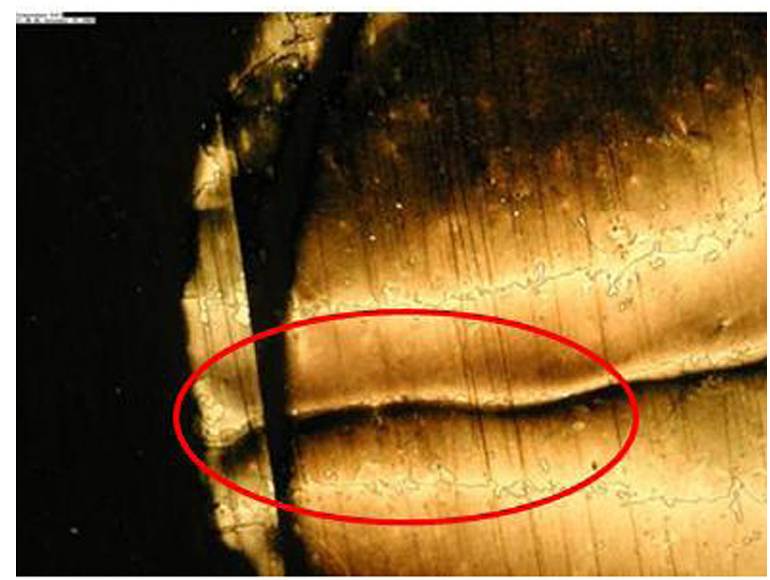

(b)

FIGURE 3 - Photos of PI-B cells after (a) 10 and (b) 15 hours of UV exposure. Photos were taken under crossed-polarizing microscope. A $5 \times$ magnification objective lens was used.

The left, middle, and right photographs of each figure represent the cells after 5, 10, and 15 hours of UV exposure, respectively. The first and second rows of each figure show the photos when the cell rubbing direction is at $0^{\circ}$ and $45^{\circ}$ with respect to the polarizer, respectively. From Fig. 2(a), both PI alignment layers are damaged within 10 hours and the degradation accumulates as the UV dosage increases. Because of the alignment layer degradation, the pretilt angle increases so that light leaks through the crossed polarizers. However, the $\mathrm{SiO}_{2}$ alignment layer [Fig. 2(b)] could withstand the UV light for more than 15 hours.

In order to see the degradation of PI alignment layer clearly, we also inspected the PI-B cell under a polarizing optical microscope. The cell rubbing direction was set parallel to the polarizer, and photos were taken after 10 and 15 hours of UV exposure. Results are shown in Figs. 3(a) and 3(b). Many lines whose axes are perpendicular to the rubbing direction appear within the illumination area. These crossed lines imply that the rubbing direction has been disturbed. As a result, the LC alignment is degraded. Figure 4 shows the photo of the $\mathrm{SiO}_{2}$ cell under microscope (magnification, $5 \times$ ) after 15 hours of UV exposure. The $\mathrm{SiO}_{2}$ align- 


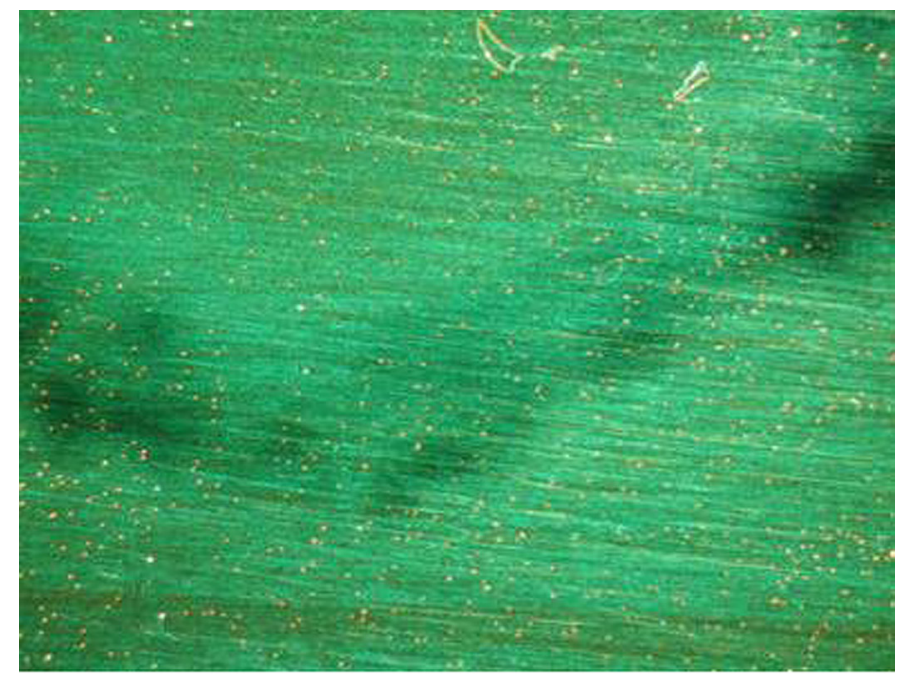

FIGURE 4 - Photograph of the $\mathrm{SiO}_{2}$ cell after 15 hours of UV exposure. The dark circle shows the edge of the illumination area. Above this line is the illumination area.

ment layers maintain excellent uniformity across the whole cell.

\section{UV stability of LC mixtures}

To examine the UV effects on LC mixtures, two commercial TFT-grade LC mixtures were investigated. They are TL-216 $\left(\Delta n=0.20\right.$ at $\lambda=633 \mathrm{~nm}$ and $\left.T=23^{\circ} \mathrm{C}\right)$ and MLC-9200-000 $(\Delta n=0.08)$. If the LC sample was exposed to the air, ${ }^{8}$ then oxidations would occur during UV exposure. In order to avoid the oxidation and humidity effects, the LC mixture was vacuum-filled into the cell. The UV illumination took place in a nitrogen chamber. We inspected the cell after each UV illumination and measured its voltage-dependent

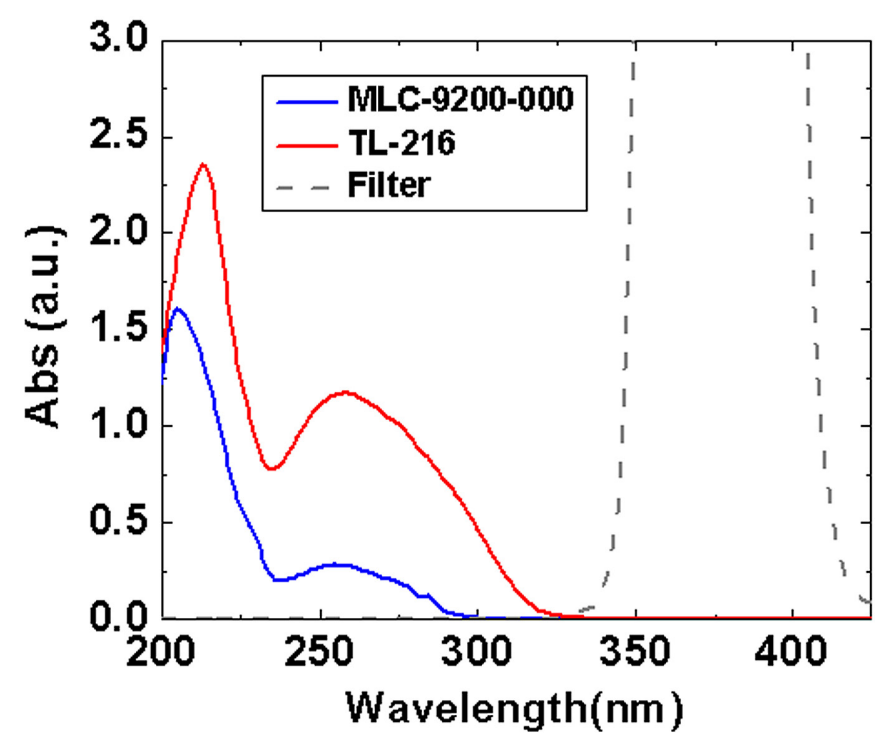

FIGURE 5 - UV absorption spectra of TL-216 (red line), MLC-9200-000 (blue line), and UV filter (gray lines). LC weight $\%=4 \times 10^{-3} \%$ in cyclohexane and cuvette gap $d=1 \mathrm{~cm}$. transmittance, birefringence, ${ }^{9}$ and visco-elastic coefficient. ${ }^{10}$ All the measurements were performed using a He-Ne laser $(\lambda=633 \mathrm{~nm})$ at $T=23^{\circ} \mathrm{C}$.

\subsection{UV absorption spectra of LC mixtures}

Two mechanisms are responsible for the observed UV degradation: structural effect and absorption effect. ${ }^{11}$ The structural effect is particularly apparent for the carbon-carbon double- and triple-bond linking groups. The LC mixtures we studied: TL-216 and MLC-9200-000 all consist of cyclohexane, phenyl, and biphenyl rings. These rings are quite stable. Thus, the absorption effect is the dominant degradation mechanism. We measured the UV absorption spectra of TL-216 (red line) and MLC-9200-000 (blue line) in a cyclohexane solvent. The weight percentage of LC mixture is $4 \times 10^{-3} \%$ and the cuvette cell gap is $1 \mathrm{~cm}$. Results are plotted in Fig. 5. The edge of transmittance of the UV filter (gray line) is also included as a reference.

The two major $\pi \rightarrow \pi^{*}$ transitions $^{12,13}$ of TL-216 appeared at $\lambda_{1} \sim 213 \mathrm{~nm}$ and $\lambda_{2} \sim 255 \mathrm{~nm}$ and the tail ends at $\sim 318 \mathrm{~nm}$. The $\pi \rightarrow \pi^{*}$ transitions of MLC-9200-000 appear at $\lambda_{1} \sim 200 \mathrm{~nm}$ and $\lambda_{2} \sim 255 \mathrm{~nm}$ and the tail ends at $\sim 300$ $\mathrm{nm}$. The similar $\lambda_{2}$ absorption peak implies that these two mixtures contain a common compound (maybe fluorinated biphenyl), but at different percentages.

\subsection{UV stability of MLC-9200-000}

We evaluated each cell's stability by characterizing its voltage-dependent transmittance $(V-T)$ curves. Figures 6(a) to 6(c) represent the $V-T$ curves of MLC-9200-000 in the $\mathrm{SiO}_{2}$, PI-A and PI-B cells, respectively. From Fig. 6(a), after 144 hours of UV exposure, the V-T curve of MLC-9200-000 in the $\mathrm{SiO}_{2}$ cell remains nearly the same as that of fresh sample. This is not surprising because MLC-9200-000 is a low birefringence LC mixture; its absorption tail is shorter than $\lambda=365 \mathrm{~nm}$. On the other hand, MLC-9200-000 in PI cells has a relatively short lifetime. The $V-T$ curves of MLC9200-000 in both PI cells start to change after 60 hours. The PI-B cell suffers an even more serious degradation. Once degradation occurs, the effective $\Delta n$ is decreased and threshold behavior smeared because of the increased pretilt angle. ${ }^{11}$

Comparing the lifetime of MLC-9200-000 in $\mathrm{SiO}_{2}$ and PI cells, we found that the PI alignment layer is less UV resistant than MLC-9200-000. Thus, it is PI layers rather than LC that limits the device lifetime. Of course, some LCs may be less stable than PI alignment layers. We have to consider the lifetime of both alignment layers and LC mixtures when evaluating the UV stability of LC devices. Whichever has the least stability determines the overall device lifetime.

From the $V-T$ curve, we calculated the LC birefringence after each UV illumination. Results are shown in Fig. 7. Here, the open squares, circles, and triangles represent the birefringence of MLC-9200-000 in $\mathrm{SiO}_{2}$, PI-A, and PI-B 

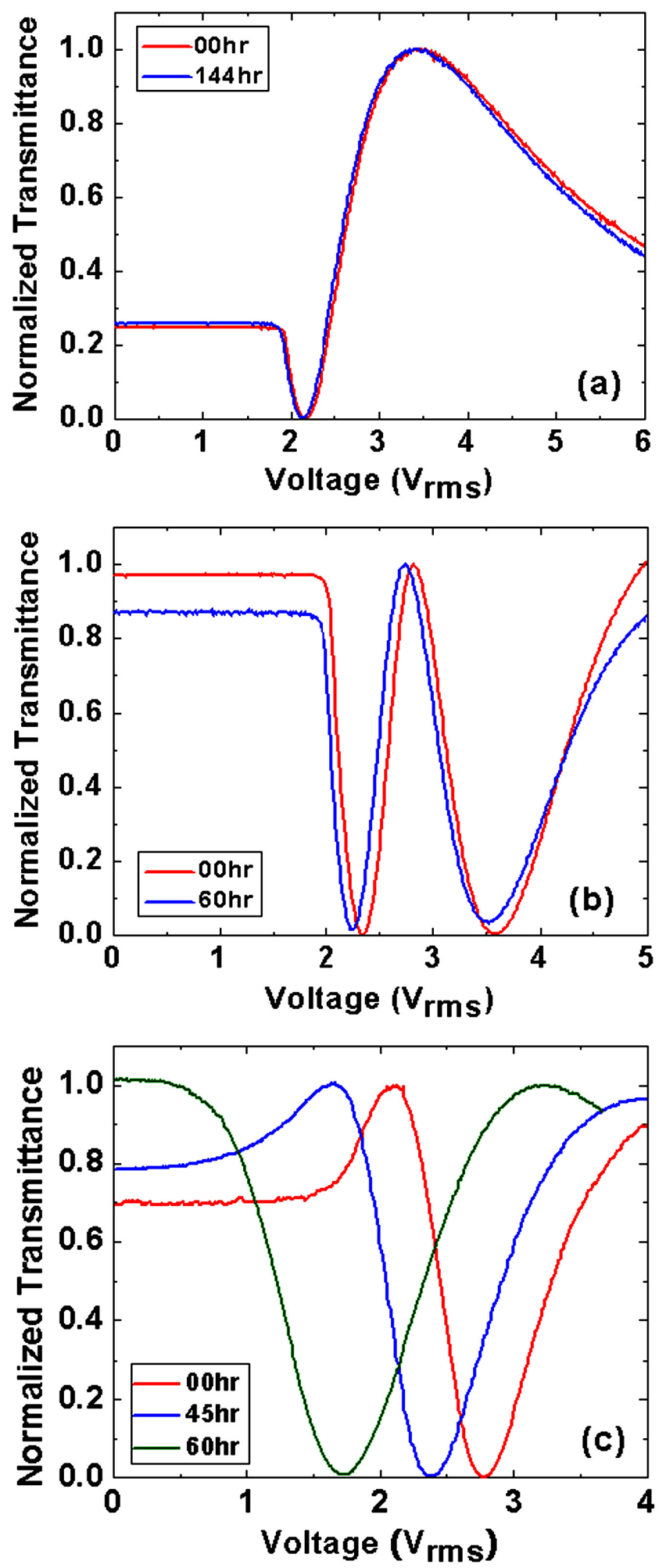

FIGURE 6 - The voltage-dependent transmittance of a (a) $\mathrm{SiO}_{2}$, (b) PI-A, and (c) PI-B alignment cell with MLC-9200-000 before (red line) and after (blue line) UV exposure. The UV intensity is $350 \mathrm{~mW} / \mathrm{cm}^{2}$.

cells, respectively. The birefringence of MLC-9200-000 in the $\mathrm{SiO}_{2}$ cell remains the same after 144 hours of UV exposure. On the other hand, the birefringence of MLC-9200000 in PI-B after 60 hours of UV exposure is decreased to

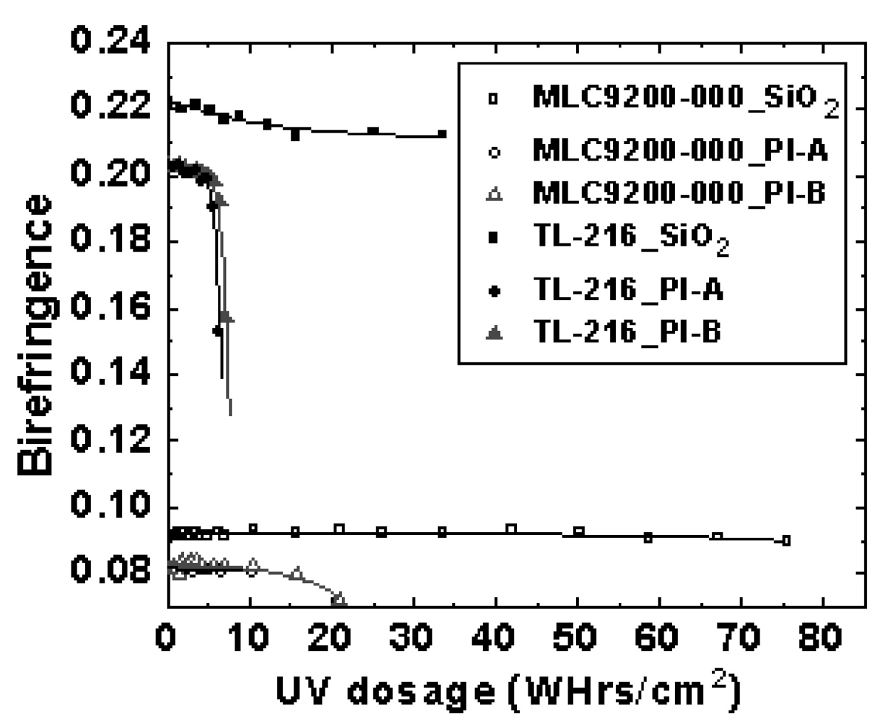

FIGURE 7 - UV exposure-time-dependent birefringence of MLC-9200-000 and TL-216 in $\mathrm{SiO}_{2}$ and PI cells. Open and filled symbols denote MLC-9200-000 and TL-216, respectively. Light source: $\lambda=365$ $\mathrm{nm}, I=350 \mathrm{~mW} / \mathrm{cm}^{2}$.

0.07. Compared to the fresh sample, this birefringence is reduced by $12.5 \%$. PI-A cell has a similar result to PI-B cell.

We also investigated how UV-induced degradation affects the visco-elastic coefficient of the LC mixture. Results are plotted in Fig. 8. Here, the open squares, circles, and triangles represent the $\gamma_{1} / K_{11}$ of MLC-9200-000 in $\mathrm{SiO}_{2}, \mathrm{PI}-\mathrm{A}$, and PI-B cells, respectively. The visco-elastic coefficient of MLC-9200-000 in the $\mathrm{SiO}_{2}$ cell remains basically unchanged after 144 hours of UV illumination. On the contrary, in both PI cells, it begins to increase after 20 hours of UV illumination. The noticeable increase in the visco-

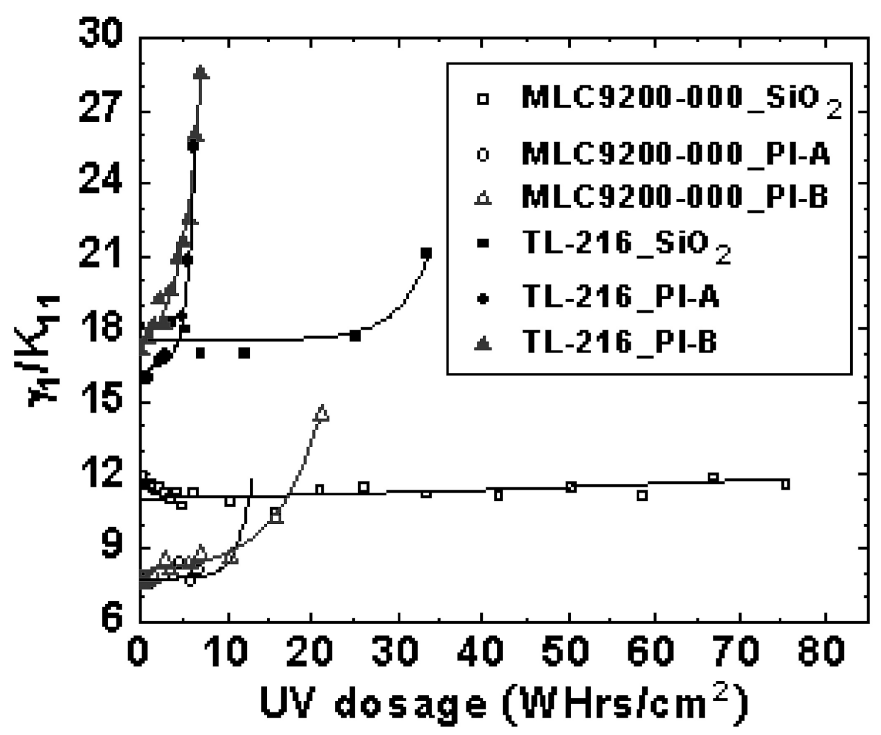

FIGURE 8 - The measured visco-elastic coefficient of MLC-9200-000 and TL-216 in $\mathrm{SiO}_{2}$ and PI cells. Open and filled symbols represent data for MLC-9200-000 and TL-216, respectively. Light source: $\lambda=365 \mathrm{~nm}$, $I=350 \mathrm{~mW} / \mathrm{cm}^{2}$. 

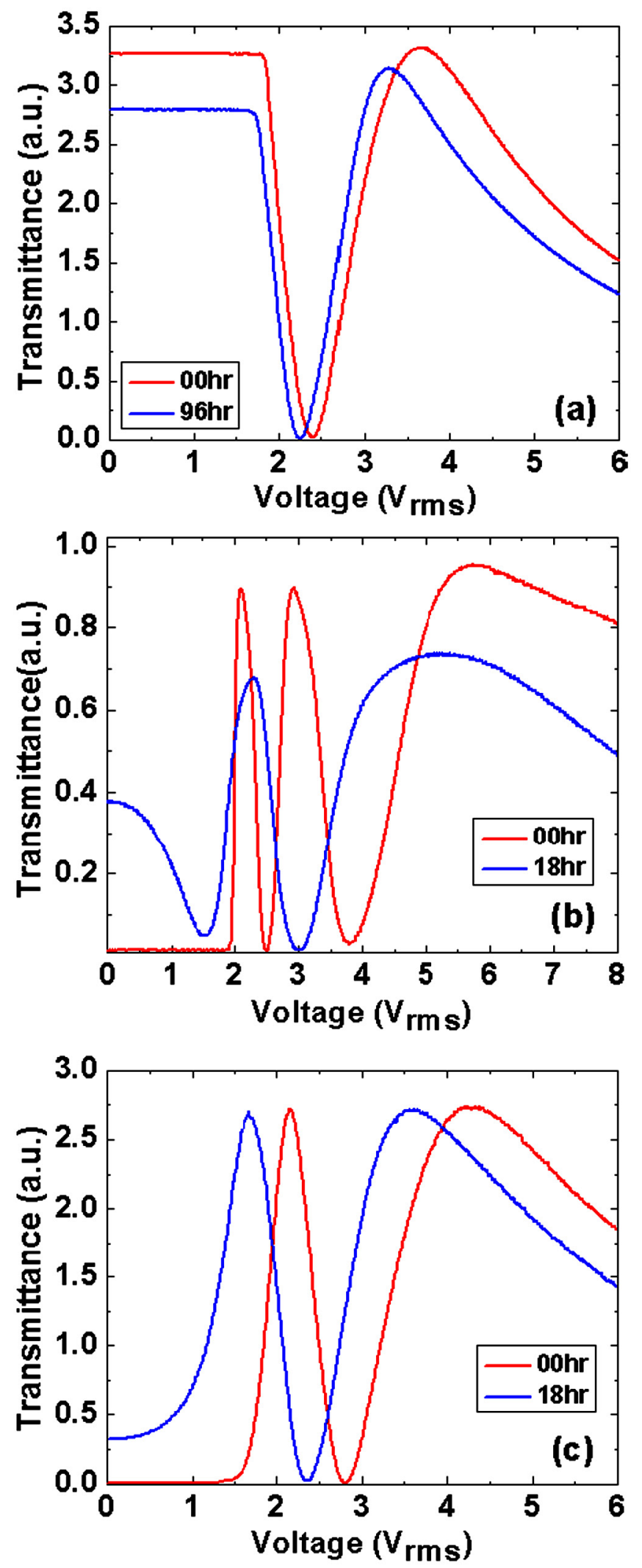

FIGURE 9 - The voltage-dependent transmittance of a (a) $\mathrm{SiO}_{2}$, (b) PI-A, and (c) PI-B alignment cell with TL-216 before (red line) and after (blue line) UV exposure. The UV intensity is $350 \mathrm{~mW} / \mathrm{cm}^{2}$.

elastic coefficient implies that the LC mixture has been seriously damaged by the UV light.

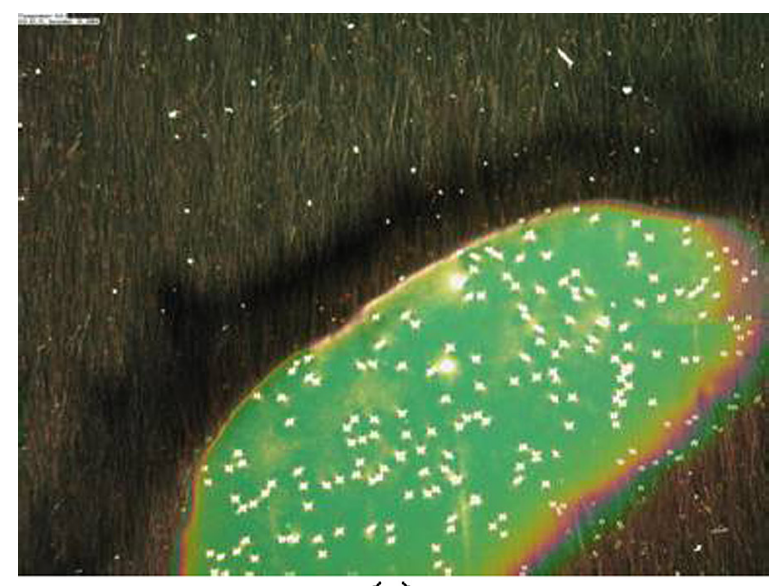

(a)

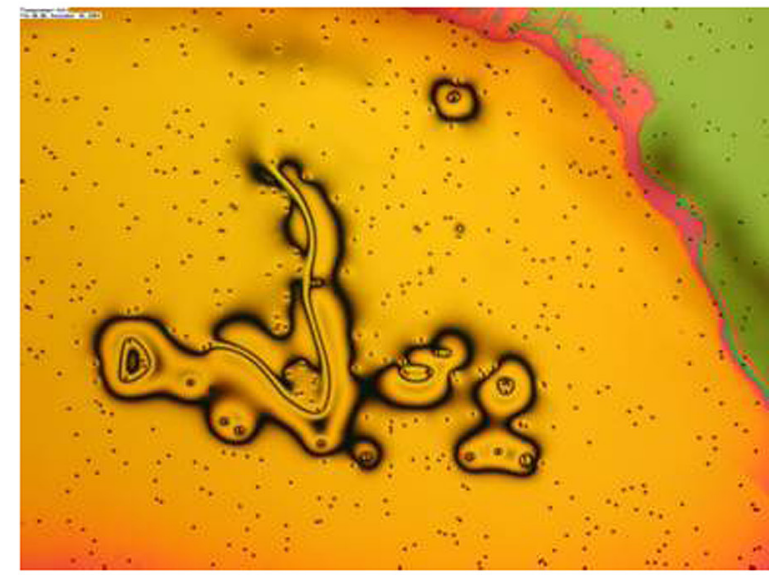

(b)

FIGURE 10 - Microscope photographs of PI-A cells filled with TL-216 after (a) 12 hours and (b) 16 hours of UV exposures. Photos (a) and (b) were taken when the cell rubbing direction was at $0^{\circ}$ and $45^{\circ}$ with respect to the polarizer, respectively. The polarizers are crossed.

\subsection{UV stability of TL-216}

Figures 9(a)-9(c) show the $V-T$ curves of TL-216 in the $\mathrm{SiO}_{2}$, PI-A, and PI-B cells, respectively. In all of the PI and $\mathrm{SiO}_{2}$ cells, the $V-T$ curves of illuminated samples were different from the fresh sample. Three common phenomena are observed as degradation gradually takes place: (1) the effective $\Delta n$ is decreased, (2) the threshold voltage is smeared and decreased, and (3) the light scattering is gradually intensified. TL-216 has a longer lifetime in the $\mathrm{SiO}_{2}$ cell than in the PI cells. The lifetime of TL-216 in the $\mathrm{SiO}_{2}$ cell and PI cell are no longer than 96 and 18 hours, respectively. The shorter lifetime of TL-216 in the PI cell comes from the unstable PI alignment layer.

It is difficult to obtain accurate $V-T$ curves of TL-216 in the $\mathrm{SiO}_{2}$ and PI cells when the cells have been illuminated continually for more than 96 or 18 hours, respectively, because defects appear at the illuminated area and the cells become non-uniform. Figure 10 shows two microscope photos of the PI-A cell filled with TL-216. We heated the cell to above clearing temperature for few minutes and let it cool 


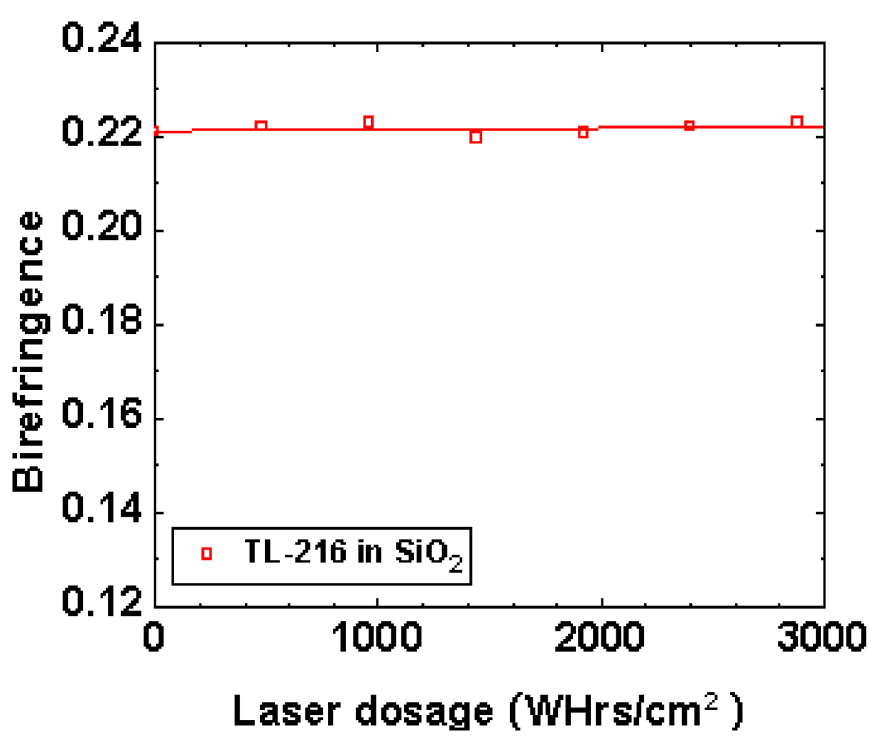

FIGURE 11 - The measured birefringence of TL-216 in $\mathrm{SiO}_{2}$ cell. Light source: He-Cd laser at $\lambda=442 \mathrm{~nm}$ and $I=20 \mathrm{~W} / \mathrm{cm}^{2}$.

down to room temperature on a polarizing optical microscope. This defected area has a non-uniform color, containing a bubble-like shape at the center of the cell. The bubble size shrinks very quickly when the temperature decreases. Meanwhile, the color inside the bubble changes continuously. From our previous studies, ${ }^{8}$ the clearing point and birefringence would decrease when the LC molecules were damaged by the UV light. Therefore, the TL-216 mixture at the center of the cell is damaged because it exhibits a different clearing temperature and birefringence from the unexposed area. From this phenomenon, we believed that the TL-216 LC mixture has a lower UV damage threshold than the PI alignment layers. Similar experiments using the inorganic $\mathrm{SiO}_{2}$ alignment layers also confirm these observations.

The birefringence of TL-216 in the PI and $\mathrm{SiO}_{2}$ cells were measured at $\lambda=633 \mathrm{~nm}$ and results are depicted in Fig. 7. The filled squares, circles, and triangles represent the birefringence of TL-216 in $\mathrm{SiO}_{2}$, PI-A, and PI-B cells, respectively. The birefringence of TL-216 in $\mathrm{SiO}_{2}$ cell after 96 hours of UV illumination is decreased by 0.01 , as compared to the fresh sample. The LC degradation was more serious in PI cell than in $\mathrm{SiO}_{2}$ cell. TL-216 in PI-A and PI-B has similar results. The birefringence after 18 hours of UV exposure drops by 0.05 , which accounts for $75 \%$ of the fresh sample. In both PI cells, bubbles formed at the edge of the illumination area after 12 hours of UV exposure.

The LC degradation affects not only birefringence but also visco-elastic coefficient. The results of the visco-elastic coefficient $\left(\gamma_{1} / \mathrm{K}_{11}\right)$ of TL-216 in PI and $\mathrm{SiO}_{2}$ cells after each UV exposure are also shown in Fig. 8. The filled squares, circles, and triangles represent the visco-elastic coefficient of TL-216 in $\mathrm{SiO}_{2}$, PI-A, and PI-B cells, respectively. The visco-elastic coefficient of TL-216 in the $\mathrm{SiO}_{2}$ cell starts to increase after 96 hours of UV illumination. Because of the degradation of the PI alignment layer, the visco-elastic coefficient of TL-216 in both PI cells increases dramatically after 18 hours of UV illumination.

\section{Stability of TL-216 under blue light}

One can argue that UV components of an arc lamp can be totally truncated by a proper filter. For a full-color LCD projector, red $(\lambda=650 \mathrm{~nm})$, green $(540 \mathrm{~nm})$, and blue (440 $\mathrm{nm}$ ) are the three primary colors. The low energy red and green photons are much less harmful than the high-energy blue photons. Thus, the ultimate photostability of a LC cell is determined by the blue light. We also studied the LC material stability at $\lambda=442 \mathrm{~nm}$. We vacuum-filled TL-216 in $\mathrm{SiO}_{2}$ cell and illuminated the test cell by using a $\mathrm{He}-\mathrm{Cd}$ laser. After every 24 hours of illumination, we monitored the LC birefringence. Results are shown in Fig. 11. From Fig. 11, no sign of degradation of TL-216 in the $\mathrm{SiO}_{2}$ cell is observed up to $2880 \mathrm{~W}$-hour/ $\mathrm{cm}^{2}$ of laser irradiation.

\section{Discussion}

The lifetime of an LC material or alignment layer could be affected by several factors, e.g., molecular structure, wavelength, and exposure intensity. For an LC material/alignment layer at a given wavelength, the degradation mechanism is not a linear function of the employed light intensity. ${ }^{14}$ The higher intensity is more harmful than the low intensity even though their total UV dosage (intensity multiplied by exposure time) remains the same. To obtain a realistic result, in principle, we should measure the lifetime at several intensities, fit the data, and extrapolate back to the intensity of the actual LCD projector. However, in our accelerated lifetime study we used a relatively high intensity $\left(I=350 \mathrm{~mW} / \mathrm{cm}^{2}\right)$ of UV light $(\lambda=365 \mathrm{~nm})$ in order to observe degradation in a reasonable exposure time. Our main objective is to compare the lifetime of different LC materials and alignment layers under the same experimental conditions.

From our experiments, the $\mathrm{SiO}_{2}$ alignment layer can withstand the longest UV exposure compared with other PI cells. We compared the lifetime of $\mathrm{LC}$ mixtures in $\mathrm{SiO}_{2}$ cells by assuming the $\mathrm{SiO}_{2}$ alignment layer was stable throughout the whole experiment. The birefringence of LC compounds is highly dependent on the conjugation length; the longer the conjugation length, the longer the absorption wavelength. Although the $\lambda_{2}$ absorption peak of MLC-9200-000 and TL-216 appears at the same wavelength and the absorption tails are far away from the cut-off UV wavelength (365 $\mathrm{nm}$ ), the lifetime of TL-216 is relatively short. From our previous study, ${ }^{8}$ we learned that the electronic transition wavelength is not the sole factor affecting the material stability. Detailed molecular structure also plays an important role. From the study of UV stability of single LC compounds, we found that the carbon-carbon double bond, triple bond, and cyano terminal group are not very stable. By avoiding these structures, it is possible to create relatively UV stable and reasonably high-birefringence LC mixtures.

Although the inorganic $\mathrm{SiO}_{2}$ alignment layer is very stable, the sputtering method is more costly because of the need of a vacuum chamber. From our experiment, MLC- 
9200-000 is very stable in the $\mathrm{SiO}_{2}$ cell up to 144 hours of UV exposure. The performance of the PI cells with MLC9200-000, however, is deteriorated severely after 60 hours of UV exposure. The degradation of the MLC-9200-000 cell is determined by the PI alignment layers. For high-brightness projection displays, we have to choose UV stable LC materials and alignment layers. In the blue laser experiments, both LC materials and alignment layers are quite robust. No sign of degradation is observed within $\sim 3000 \mathrm{~W}-$ hour $/ \mathrm{cm}^{2}$ of continuous irradiation. This implies if we can filter out any residual UV components from the arc lamp, the LCD projector will have a very long lifetime.

\section{Conclusion}

The UV and blue stability of PI and $\mathrm{SiO}_{2}$ cells and LC mixtures (MLC-9200-000 and TL-216) were studied. Experimental results show the $\mathrm{SiO}_{2}$ cells are much more stable than PI cells. The stability of the alignment layer is a critical factor affecting the stability of the entire device. Moreover, the UV stability of LC mixture depends not only on the electronic transition wavelength, but also on the molecular structures. From our study, although the high-birefringence LC mixture is less stable than the low-birefringence mixture under UV exposure, it is quite stable under blue laser $(\lambda=$ $442 \mathrm{~nm}$ ) irradiation. To ensure a long-lasting projection display, not only a longer cutoff-wavelength blue filter but also UV-resistant inorganic or organic alignment layers and LC mixtures should be carefully chosen.

\section{Acknowledgments}

The authors would like to thank Toppoly Optoelectronics (Taiwan) for the financial supports.

\section{References}

1 A M Lackner, J D Margerum, L J Miller, and W H Smith, Jr, "Near ultraviolet photostability of liquid-crystal mixtures," Mol Cryst Liq Cryst 141, 289-310 (1986).

2 J L West, X Wang, Y Ji, and J R Kelly, "Polarized UV-exposed polyimide films for liquid crystal alignment," SID Symposium Digest Tech Papers 26, 703-705 (1995).

3 H Barna, M Lu, M Samant, C Cai, and A Rosenbluth, "Liquid crystal alignment degradation induced by photo-irradiation," SID Symposium Digest Tech Papers 32, 980-983 (2001).

4 W Oepts, E Ito, M Reijme, A Verschueren, and E Alexander, "Degradation of liquid crystal alignment upon high intensity illumination in microdisplays," Eurodisplay, 201-204 (2002).

5 R D Sterling and W P Bleha, "D-ILA technology for electronic cinema," SID Symposium Digest Tech Papers 31, 310-313 (2000).

6 S Gauza, C H Wen, B J Tan, and S T Wu, "UV stable high birefringence liquid crystals," Jpn J Appl Phys 43, 7176-7180 (2004).

$7 \mathrm{P}$ Janssen, J A Shimizu, J Dean, and R Albu, "Design aspects of a scrolling color LCOS display," Displays 23, 99-108 (2002).

$8 \mathrm{C} \mathrm{H}$ Wen, S Gauza, and S T Wu, "Ultraviolet stability of liquid crystals containing cyano and isothiocyanato terminal groups," Liq Cryst 31, 1479-1485 (2004).

$9 \mathrm{~S} \mathrm{~T} \mathrm{Wu}, \mathrm{U}$ Efron, and L D Hess, "Birefringence measurements of liquid crystals," Appl Opt 23, 3911-3915 (1984).

10 S T Wu and C S Wu, "Experimental confirmation of Osipov-Terentjev theory on the viscosity of nematic liquid crystals," Phys Rev A $\mathbf{4 2}$, 2219-2227 (1990).
$11 \mathrm{~S} \mathrm{~T} \mathrm{Wu}$, "Molecular design strategies for high birefringence liquid crystals," Mater Res Soc Symp Proc 709, 219-228 (2002).

$12 \mathrm{~S} \mathrm{~T} \mathrm{Wu}$, "Birefringence dispersions of liquid crystals," Phys Rev A 33 , 1270-1274 (1986).

$13 \mathrm{~S} \mathrm{~T} \mathrm{Wu}, \mathrm{C} \mathrm{S} \mathrm{Wu}, \mathrm{M}$ Warenghem, and M Ismaili, "Refractive-index dispersions of liquid-crystals," Opt Eng 32, 1775-1780 (1993).

14 S Yakovenko, V Konovalov, and M Brennesholtz, "Lifetime of single panel LCOS imagers," SID Symposium Digest Tech Papers 35, 64-67 (2004).

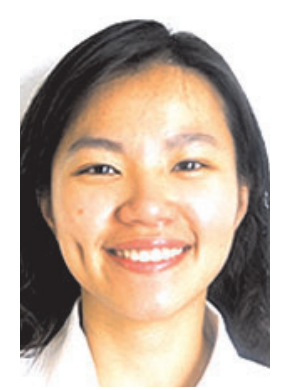

Chien-Hui Wen received her B.S degree in applied chemistry from National Chiao Tung University, Taiwan, in 2001 and her M.S. degree in optics from the University of Central Florida, Orlando, Florida in 2003. She is currently a Ph.D. candidate at College of Optics and Photonics/CREOL/FPCE, University of Central Florida, Orlando, Florida. Her current research is to develop fast-response negative dielectric anisotropy and dual-frequency liquid-crystal (LC) materials and study their performance. These materials have wide application in visible and infrared regions, such as TV and laser-beam steering.

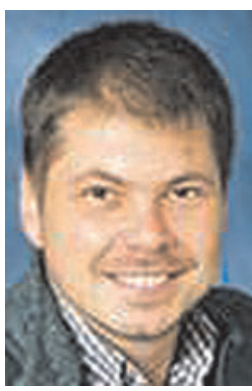

Sebastian Gauza received his Ph.D. in chemistry from Military University of Technology in Warsaw, Poland in 2001. He is currently a research scientist at College of Optics and Photonics/CREOL/FPCE, University of Central Florida, Orlando, Florida. His current research is to develop novel high-birefringence nematic liquid-crystal single compounds and mixtures for photonic applications. His future work will focus on novel nematic compositions for laser-beam steering systems.

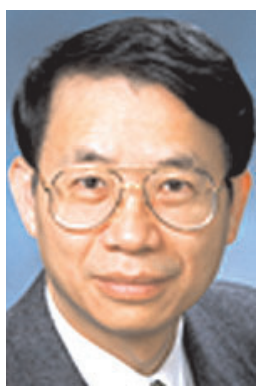

Shin-Tson Wu is a PREP professor at the College of Optics and Photonics, University of Central Florida. Prior to joining UCF in 2001, Dr. Wu worked at Hughes Research Laboratories (Malibu, California) for 18 years. He received his Ph.D. from the University of Southern California and his B.S. in physics from National Taiwan University. His studies at UCF concentrate on foveated imaging, bio-photonics, optical communications, liquid-crystal displays, and liquid-crystal materials. Dr. Wu is a Fellow of the IEEE, SID, and OSA. Prof. Wu has co-authored two books: Reflective Liquid Crystal Displays (Wiley, 2001) and Optics and Nonlinear Optics of Liquid Crystals (World Scientific, 1993), four book chapters, and over 220 journal papers. He holds 22 patents. 